\title{
Effects of an ACTH 4-9 Related Peptide Upon Intracranial Self Stimulation and General Activity in the Rat
}

\author{
Richard J. Katz \\ Mental Health Research Institute, Department of Psychiatry, University of Michigan Medical Center, Ann Arbor, Michigan 48109, USA
}

\begin{abstract}
Adult male Sprague-Dawley rats were stereotactically implanted with electrodes within the anterior medial forebrain bundle. The rats were trained to respond for intracranial self-stimulation (ICS) and treated with control solution or varying doses of an ACTH 4-9 related synthetic peptide (Org 2766; $\mathrm{H}$ $\operatorname{Met}\left(\mathrm{O}_{2}\right)$-Glu-His-Phe-D-Lys-Phe-OH). The drug affected ICS as measured in overnight response records, with the highest dose reliably increasing the amount of responding. In a second experiment rats were similarly treated and general activity was assessed. No remarkable changes in activity were present at any tested dose. The findings corroborate previous reports suggesting ACTH-related peptides may be active in a variety of motivated tasks, but less active with respect to general activity.
\end{abstract}

Key words: Activity - ACTH 4-9 - Neuropeptides - Reinforcement - Self stimulation

Fragments of adrenocorticotrophic hormone (ACTH) without intrinsic adrenal stimulating effects may nonetheless affect behavior. ACTH 4-10, the D-Phe analog of ACTH 4-10, and the structurally modified analog of ACTH 4-9, Organon 2766 [Org 2766; H-Met $\left(\mathrm{O}_{2}\right)$ Glu-His-Phe-D-Lys-Phe-OH] may affect attention and memory related processes (for reviews see Sandman et al. 1976; Van Reizen et al. 1977) and also modify responses to pain and the normal occurrence of murine grooming (Gispen et al. 1975, 1976).

The variety of psychoactive properties of ACTHrelated peptides might argue for significance in many motivated behaviors. The present report is an examination of the potential influence of Org 2766 upon intracranial self-stimulation (ICS) and general activity. This peptide is of particular interest given previous indications of a significant (up to $1,000 \times$ ) increase in pharmacological potency in comparison with ACTH 4-10, allowing the use of systemic injections of the analog. The peptide is known to possess some (e.g., antiamnestic; Rigter et al. 1976) but not all (e.g., not excessive grooming; Gispen et al. 1975) behavioral effects of other ACTH-related peptide fragments.

The self-stimulation task allows further assessment of neuropeptide influences upon a novel form of motivated behavior. No other published studies to date have examined the effects of ACTH fragments on ICS. Bohus (1979) notes an as yet unpublished study by Nyakos indicating some degree of ICS facilitation after ACTH 4-10. However, since neither rates nor doses are provided, this report is difficult to interpret. The first experiment examined ICS while a separate experiment assessed any effects upon a more general measure of activity, home cage activity.

\section{Materials and Methods}

Subjects. Seventy-nine adult male Sprague-Dawley rats (49 for experiment 1, 30 for experiment 2) (Charles River) ranging in weight from $380-400 \mathrm{~g}$ were individually maintained with food (Teklad $4.0 \%$ fat rodent diet S-0836) and tap water continuously available. $12 \mathrm{~h}$ light/dark cycles were automatically programmed, with lights on between 7 a.m. and 7 p.m.

Apparatus. Detailed descriptions of both apparatus and surgery have been published elsewhere (Katz et al. 1978; Katz 1979). All testing was carried out in the subject's home cages. For Experiment 1, normal $25 \times 18 \times 17 \mathrm{~cm}$ rack mounted stainless steel cages were modified to house overhead mounted $14 \times 16 \mathrm{~cm}$ stainless steel plates, which served as both manipulanda and contacts for stimulation. Contact with the plate by the rat's head-mounted brushing displaced the plate upwards, closing a microswitch with a $25 \mathrm{~g}$ operating requirement. This allowed circuit completion and delivery of a $0.3 \mathrm{~s}$ train of $60 \mathrm{~Hz}$ sinusoidal current through the plate to the brushing, and to an attached intracranial electrode. Normally a fully extended rearing response was necessary to move the plate sufficiently to complete the circuit. Circuit completion was achieved with the floor acting as the return circuit for stimulation. This arrangement permitted continuous ad lib. access to stimulation. A series of 
capacitors and resistances, and a zero crossing relay were used to assure constant current stimulation within and across stimulation trains.

For Experiment 2, rats were individually maintained in $51 \times 41$ $\times 22 \mathrm{~cm}$ polypropylene containers which rested upon remote sensing devices (Stoelting, Chicago, Ill.) tuned to equivalent sensitivity. The sensing devices operated through the disruption of tuned oscillators.

Surgery. Surgical details (Experiment 1) are essentially similar to previous descriptions. Rats were anesthetized with $35 \mathrm{mg} / \mathrm{kg}$ of sodium pentobarbital and stereotactically implanted with a single $0.025 \mathrm{~cm}$ diameter nichrome wire electrode insulated to the tip. Electrodes were aimed at the anterior aspect of the medial forebrain bundle (anterior-posterior, medial-lateral, and dorsal-ventral coordinates in $\mathrm{mm}$ from Bregma, using a level skull $=0.0,2.0,-8.0$ ) and were attached to a brass brush taken from a commercially available suede brush, as previously described. The brush electrode assembly was attached to the skull with five stainless steel screws and acrylic dental cement (Katz 1979). Histological examination at the close of testing was by the method of Hosko (1975) using wet cryostatically sectioned tissue at $40 \mu$ as a photomicrographic negative.

Behavioral Procedure. In Experiment 1 rats were allowed 1 week to recover from surgery prior to being exposed to any current. Current was then uniformly activated at an intensity of $50 \mu \mathrm{A}$, and levels were adjusted over the next 7 days to maintain rates of stimulation between 1,200 and 4,500 responses over a $24 \mathrm{~h}$ recording interval. Normal rates of responses without current were consistently less than 50 responses per night. Final current values ranged between 20 and $80 \mu \mathrm{A}$. For all subjects greater than $95 \%$ of responses occurred during the dark phase of the lighting cycle. Stimulation parameters remained unchanged for individual subjects for the remainder of testing. All but three rats acquired the panel press response spontaneously during normal exploration of the apparatus. The remaining rats were trained using standard operant procedures. All data were acquired using BSR/LVE 12V logic modules interfaced with a Burpee paper tape punch. A similar period of undisturbed (recovery) activity was also permitted for the rats in Experiment 2.

Drugs. Drugs were prepared in polypropylene containers immediately prior to use. Injections of vehicle $(0.9 \%$ sodium chloride solution) or experimental compound (Org $27660,5,10$, or $20 \mu \mathrm{g} / \mathrm{kg}$ in Experiment $1 ; 0,10,20 \mu \mathrm{g} / \mathrm{kg}$ in Experiment 2) were administered IP, $1 \mathrm{ml} / \mathrm{kg}$. All rats initially received two treatments, the first of which was vehicle and the second of which was one of the three drug treatments or vehicle. All injections were administered $20 \mathrm{~min}$ prior to the onset of the normal dark cycle. Doses were within the range established by previous studies on this peptide (Rigter et al. 1976).

Statistical Procedure. Two scores were recorded for each subject, a day 1 score (vehicle) and a day 2 score (vehicle or drug). All data are presented as means and standard errors based upon overnight response records (i.e, $12 \mathrm{~h}$ sessions). A covariance procedure (univariate analysis of covariance; Wildt and Ahtola 1978) was used to assess significance of groups, controlling for basal differences in level of responding to vehicle. Analysis of covariance has been suggested as an alternative to more traditional analysis of variance of percentage transformed data, is more defensible upon statistical grounds (Anderson and Lydic 1977), and is considered a more sensitive indicator of main effects. It should be noted that all assumptions for analysis of variance models are also required for covariance analysis. Sheffe allowances were used for post-hoc comparisons across groups.

\section{Experiment 1}

As noted, the initial experiment examined the effects of the ACTH 4-9 analog upon rats with chronic access to

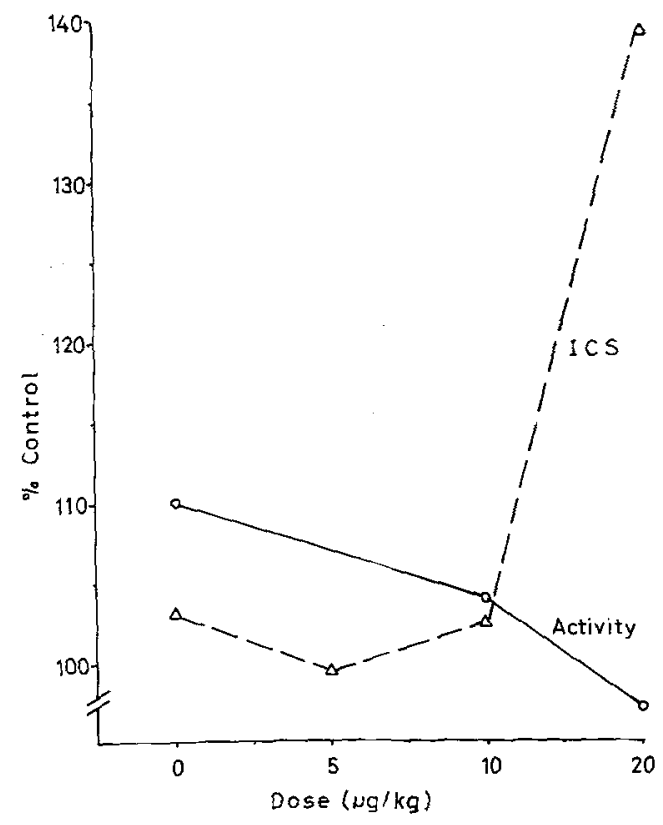

Fig. 1. Summary of percentage increases due to an ACTH 4-9-related peptide in two behavioral tasks. Self-stimulation is presented as the broken line, and general activity as the solid line. For self-stimulation control values upon which comparisons were based scores were; $5,220 \pm 930,7,700 \pm 1,620,3,420 \pm 570 ;$ and $4,200 \pm 780$ (for 0,5 , 10 , and $20 \mu \mathrm{g} / \mathrm{kg}$ of $\mathrm{Org} 2766$ respectively). For the activity measure baseline untransformed scores were $18,709 \pm 1,201,20,056 \pm 2,192$, and $17,886 \pm 2,015$ (for 0,10 , and $20 \mu \mathrm{g} / \mathrm{kg}$ )

intracranial reward. Should the peptide affect motivated behaviors, then it is possible this would be reflected in increased responding for ICS.

\section{Methods}

Methods and procedures are identical to previous descriptions.

\section{Results}

Peptide injection was followed by a facilitation of intracranial-reinforcement at $20 \mu \mathrm{g} / \mathrm{kg}$ for the majority of rats tested, and this proved to be statistically significant. Data are summarized in Fig. 1. At the outset it should be noted that the slopes of the regression lines did not differ across dosage conditions ( $F 3,41=1.2, P=0.3$ ), thus the main condition for carrying out further analysis was satisfied. An overall groups effect was present across cells $(F 1,44=304.9$, $P<0.0001)$ and this remained present when baseline differences were accounted for $(F 3,44=2.8$, $P<0.05)$. Examination of cells using a multiple comparison procedure indicated a significant $(F=5.2$, $P<0.025)$ differences between vehicle and the highest dose, and essential equivalence of remaining cells ( $F$ 's 


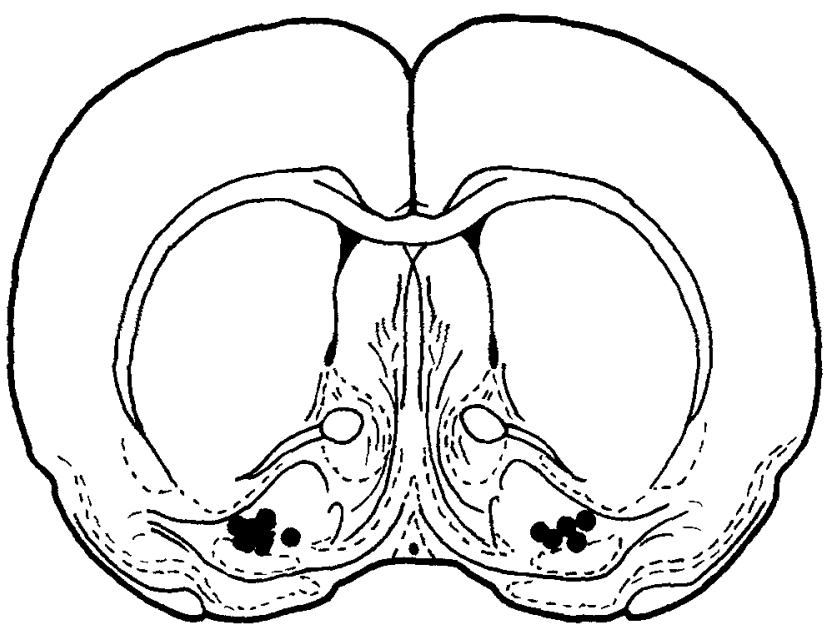

Fig. 2. Composite histology for self-stimulation sites in experiment 1. Fifteen rats were chosen at random from all groups and sacrificed with an overdose of sodium pentobarbital. Visualization of sites was by the method of Hosko as described in text

for vehicle versus lower doses $=0.4,0.2, P \simeq 0.5$ ). The lower doses were significantly different from the higher dose $(F=6.7,4.5$, respectively, $P<0.05)$. It may be concluded only the highest dose of peptide was effective in a statistically reliable manner under the present circumstances.

Histological examination of a random sample of 15 rats at the close of testing indicated that electrode sites were located within the medial forebrain bundle at its anterior tip. Results are preserved graphically (Fig. 2). No apparent relationship between specific electrode locus and degree of facilitation was present, although it should be noted clustering of electrodes may have limited the anatomic variability in the sample.

\section{Experiment 2}

The results of Experiment 1 indicated a reliable effect of the peptide upon brain stimulation. The generality of this activity effect may be questioned. To address this issue the effects of the peptide upon general activity were further examined.

\section{Methods}

Materials and methods are identical to previous de scription.

\section{Results}

No consistent effects of ACTH 4-9-related peptide injection upon general activity were found. Data are summarized in Fig. 1. While regression slopes were not significantly different $(F 2,24=2.6, P \simeq 0.1)$, as is required for the analysis, and although a groups effect across cells was present $(F 1,26=7.3, P<0.01)$, this was not due to drug conditions $(F 2,26=0.2)$ but represented a significant relation of day 1 , day 2 scores across the subjects and cells. Sheffe comparisons indicated all $F$ ratios for comparisons across drug conditions (vehicle vs. 10 or $20 \mu \mathrm{g} / \mathrm{kg}$ ) to be less than 1.0 .

\section{Discussion}

In experiment 1 an ACTH 4-9-related peptide reliably increased ICS responding in the rat at the highest dose tested. Thus it was active, more closely resembling previous reports from Rigter et al. (1976) than of Gispen et al. (1975). One interpretation of this rests with a sensitization of the neural systems subserving brain stimulation. The net result of this sensitization process would be an increase in the reinforcing properties of stimulation. This is not the only possible interpretation, however. Another possible explanation may be that Org 2766 decreased the aversiveness of stimulation. Perhaps the electrode sites were of a motivationally mixed character (i.e., were both reinforcing and somewhat painful) and the experimental compound was analgesic. The reinforcing properties of stimulation might have remained constant but aversion might have been less after treatment. While Org 2766 and related ACTH fragments are to a degree analgesic (Walker 1979), this is only after central injection. We know of no data supporting analgesic properties for systemically administered Org 2766 . Also since the selfstimulation sites in this experiment required no priming, and supported ICS behavior for several weeks, there are only limited grounds for assuming any aversive characteristics of stimulation. By a third explanation, this particular peptide may have enhanced attention. Perhaps rats were more aroused and therefore more ready to engage in all activity, including selfstimulation. If this were so it would of necessity be due to a very specific form of arousal, since general activity was not affected by the peptide. Other reports (e.g., Wolthius and DeWeid 1976) also suggest that ACTH analogs may have attentional effects not readily seen in general activity. Jouhaneau-Bowers and Le Magnen (1979) have demonstrated that ACTH 1-24 and ACTH $4-10$ are positively reinforcing in a selfadministration task. The present demonstration corrobates this observation using a novel peptide and method.

It is not our intention to claim that 2766 is identical in all respects with other ACTH fragments. ACTH fragments, particularly those with chemical substitutions may have a variety of activities not present in the parent molecule, and vice versa (Van Riezen et al. 1977). Thus, there are only limited grounds for extending these findings to other fragments. It might be 
argued that some fragments do affect reinforcement in both this and other designs, and that the present results are consistent with the compounds possessing direct or indirect activity within central motivational systems.

Acknowledgements. Organon 2766 was generously donated by Organon Corporation, Oss, The Netherlands. Giulio Baldrighi provided excellent technical assistance throughout all phases of the experiment. Bob Shea provided statistical assistance. R. J. Katz is a sloan Foundation Fellow in neuroscience.

\section{References}

Anderson D, Lydic R (1977) Ratio data and the quantification of drug effects. Biobehav Rev 1:55-57

Bohus B (1979) Current review: Effects of ACTH-like neuropeptides on animal behavior and man. Pharmacology 18:113-122

Gispen WH, Buitelaar J, Wiegant VM, Terenius L, DeWied D (1976) Interaction between ACTH fragments, brain opiate receptors and morphine induced analgesia. Eur J Pharmacol 39:393-397

Gispen WH, Wiegant VM, Greven HM, DeWied D (1975) The induction of excessive grooming in the rat by intraventricular application of peptides derived from ACTH-structure activity studies. Life Sci 17:645-652
Hosko MJ (1975) Technique for rapid permanent documentation of intracerebral electrode sites. Physiol Behav 14:367-368

Jouhaneau-Bowers M, Le Magnen J (1979) ACTH self administration in rats. Pharmacol Biochem Behav 10:325-328

Katz RJ (1979) The temporal structure of reinforcement: An analysis of brain stimulated reward. Behav Neural Biol 26:416-430

Katz RJ, Baldrighi G, Roth KA (1978) Appetitive determinants of self stimulation. Behav Biol 23:500-508

Rigter H, Janssens-Elbertse R, Van Riezen H (1976) Reversal of amnesia by an orally active ACTH 4-9 analog (Org 2766). Pharmacol Biochem Behav (supp: The peptides) 5:53-58

Sandman CA, Miller LH, Kastin AJ (1976) The neuropeptides: Pharmacology, physiological substrates, and behavioral effects. Pharmcol Biochem Behav (supp) $5: 1-3$

Van Riezen H, Rigter H, DeWied D (1977) Possible significance of ACTH fragments for human mental performance. Behav Biol $20: 311-324$

Walker JM (1979) ACTH and central mechanisms of pain inhibition. Doctoral dissertation. Ohio State University

Wildt AR, Ahtola OT (1978) Analysis of covariance. Sage, Beverly Hills, CA

Wolthius OL, DeWeid D (1976) The effect of ACTH analogues on motor behavior and visual evoked responses in the rat. Pharmacol Biochem Behav 4:273-278

Received November 15, 1979; Final version May 15, 1980 